\section{Pancreatic cystic lymphangioma in a 6-year-old girl, diagnosed by endoscopic ultrasound (EUS) fine needle aspiration}
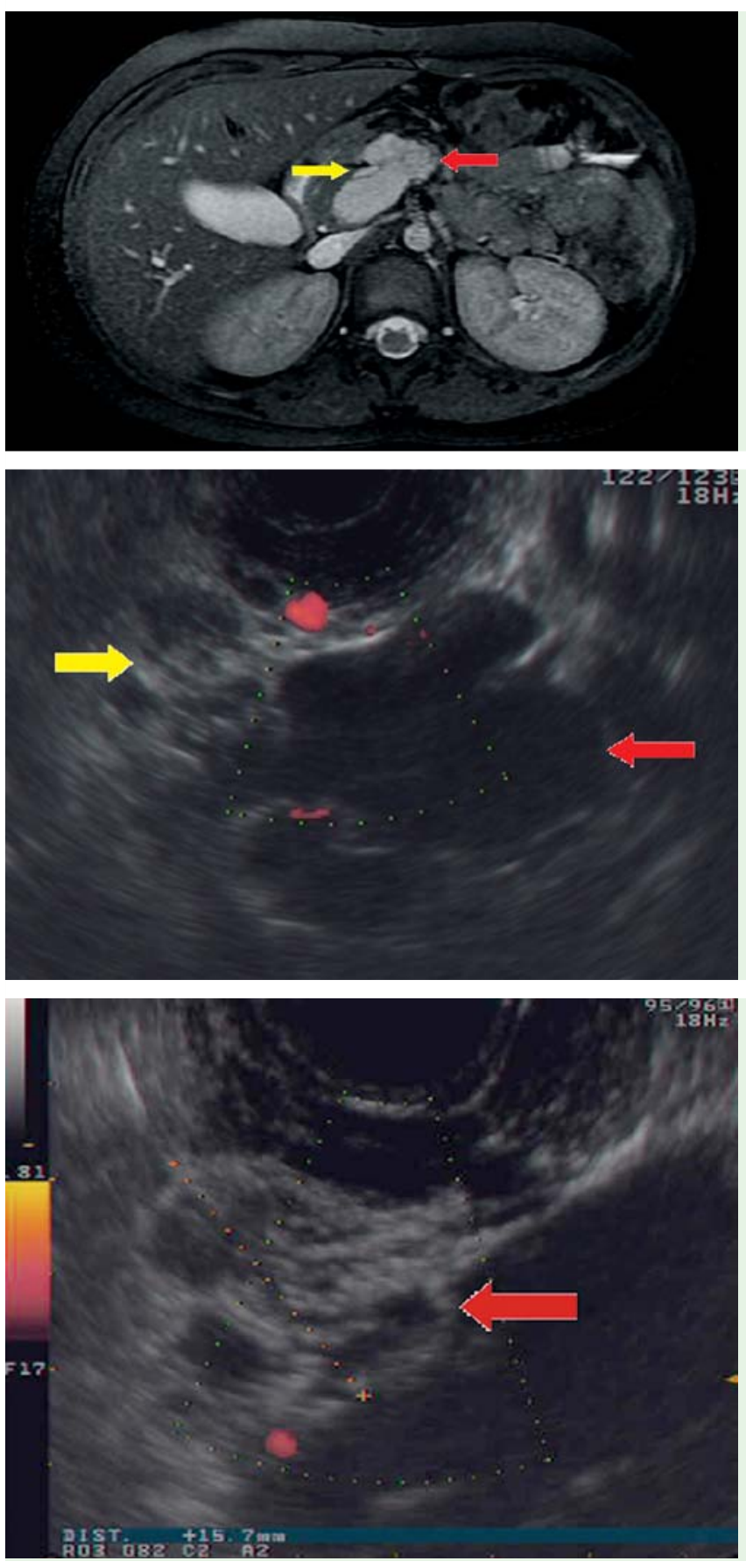

Fig. 1 Magnetic resonance (MR) image of the pancreatic cystic lesion (yellow arrow, mesenteric vein; red arrow, suspected solid area).

Fig. 2 Endoscopic ultrasound (EUS) view of the pancreatic cystic lesion (yellow arrow, microcystic area; red arrow, macrocystic area).

Fig. 3 Endoscopic ultrasound (EUS) view of the microcystic area (arrow).
Pancreatic cystic lesions are challenging clinically because they represent a spectrum of different lesions, ranging from benign to malignant. At times, the final diagnosis is made only at surgery. We report a final diagnosis of a pancreatic cystic lymphangioma, made using endoscopic ultrasound fine needle aspiration (EUSFNA) in a young girl, with cytological examination and measurement of the level of triglycerides in the intracystic fluid.

A 6-year-old girl showed evidence of a pancreatic head cystic lesion on transabdominal ultrasonography. Magnetic resonance imaging (MRI) showed a multilobular cystic lesion, with an inverted $C$ shape, around the splenomesenteric confluence (๑ Fig. 1).

The MRI also showed a small, irregular area, which was suspected of being a solid component within the lesion. Endosonography with linear array showed a micromacrocystic lesion, $4 \mathrm{~cm}$ in diameter, in the pancreatic head and uncinate process (๑ Figs. 2, 3).

No solid mass was seen. EUS-FNA with a $22 \mathrm{G}$ needle was carried out to evacuate the lesion. The intracystic fluid appeared milky and viscous ( $\bullet$ Fig. 4).

Intracystic fluid analysis showed amylase/ lipase 200/1720 U/L, carcinoembryonic antigen (CEA) $0.2 \mathrm{ng} / \mathrm{mL}$, and triglycerides $10570 \mathrm{mg} / \mathrm{dL}$. Cytology showed normal lymphocytes. The final diagnosis was pancreatic cystic lymphangioma. Abdominal ultrasound confirmed the presence of an unchanged lesion at 1 year follow-up and the patient remains asymptomatic.

Cystic lymphangioma of the pancreas is an extremely rare, benign tumor of lymphatic origin $[1,2]$. Possible locations are in the retroperitoneum, within or outside the pancreas [3]. Histologically, it appears as a polycystic lesion, with the cysts separated by thin septa, and lined with endothelial cells. It can be difficult to distinguish this lesion from other pancreatic cystic lesions. A final diagnosis is often achievable only by histopathological examination of the resected lesion [1 - 3]. In cases of pancreatic cystic lymphangioma, EUS-FNA with cytological examination and measurement of the level of triglycerides in the intracystic fluid can provide a safe and accurate diagnosis $[4,5]$. 


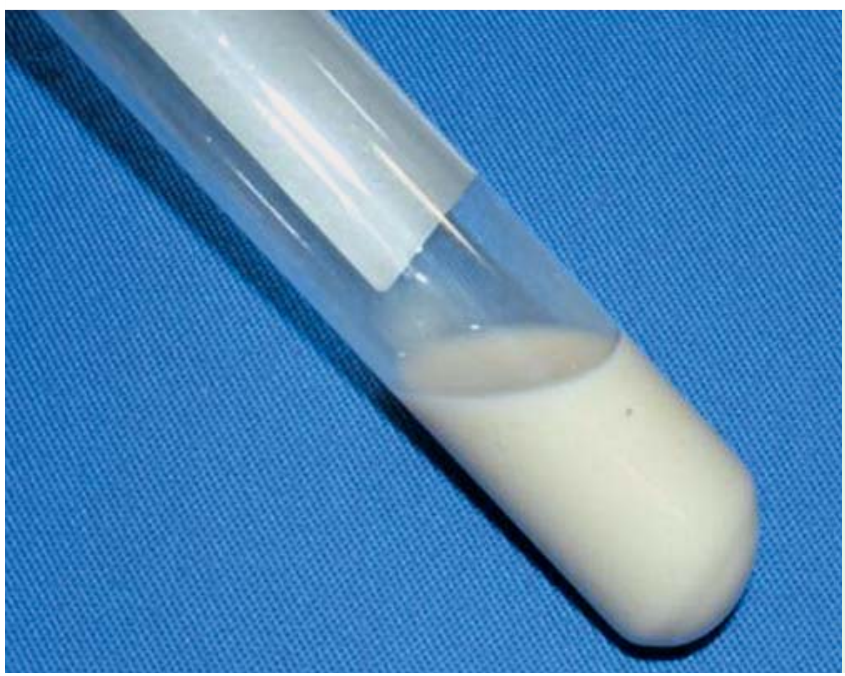

Fig. 4 The intracystic fluid.

\author{
Endoscopy_UCTN_Code_CCL_1AF_2AZ_3AD
}

\section{Competing interests: None}

\section{Barresi ${ }^{1}$, I. Tarantino ${ }^{1}$, G. Curcio ${ }^{1}$, F. Mocciaro' ${ }^{1}$, P. Catalano ${ }^{2}$, M. Spada ${ }^{2}$, M. Traina ${ }^{1}$}

1 Gastroenterology and Endoscopy Unit, ISMETT, Palermo, Italy

2 Pediatric Surgery and Transplantation Department, ISMETT, Palermo, Italy

\section{Bibliography}

DOI $10.1055 / \mathrm{s}-0030-1256079$

Endoscopy 2011; 43: E61 - E62

(c) Georg Thieme Verlag KG Stuttgart · New York . ISSN 0013-726X

\section{Corresponding author}

\section{Dr. L. Barresi}

Gastroenterology and Endoscopy Unit ISMETT

Via Tricomi 1

Palermo

Italy

Fax: +39-091-2192288

Ibarresi@ismett.edu 